\title{
Boundary layer stabilization using free-stream vortices
}

\author{
L. Siconolfi ${ }^{1}$, S. Camarri ${ }^{1} \dagger$ and J. H. M. Fransson ${ }^{2}$ \\ ${ }^{1}$ Dipartimento di Ingegneria Civile ed Industriale, Università di Pisa, I-561 26 Pisa, Italy \\ ${ }^{2}$ Linné Flow Centre, KTH-Royal Institute of Technology, SE-100 44 Stockholm, Sweden
}

(Received ?; revised ?; accepted ?.)

In this numerical investigation we explore the possibility to apply free-stream vortices as a passive flow control method for delaying transition to turbulence delay. The work is motivated by previous experimental studies demonstrating that stable streamwise boundarylayer (BL) streaks can attenuate both two- and three-dimensional disturbances inside the BL leading to transition delay, with the implication of reducing skin-friction drag. To date, successful control has been obtained using physical BL modulators mounted on the surface in order to generate the stable streaks. However, surface mounted BL modulators are doomed to fail when the BL is subject to free-stream turbulence (FST), since a destructive interaction between the two is inevitable. In order to tackle free-stream disturbances, such as FST, a smooth surface is desired, which has motivated us to seek new methods to induce streamwise streaks inside the boundary layer. A first step, in a systematic order, is taken in the present paper to prove the control idea of generating free-stream streamwise vortices for attenuating ordinary Tollmien-Schlichting waves inside the boundary layer. In this proof-of-concept study we show that, by applying a spanwise array of ideal counter-rotating free-stream vortices, inducing streamwise BL streaks further downstream, it is possible to alter the BL stability characteristics to such degree that transition delay may be accomplished. For the demonstration we use direct numerical simulations along with biglobal stability analysis.

Key words: Boundary layer control $\cdot$ transition delay $\cdot$ boundary layer stability . Tollmien-Schlichting waves

\section{Introduction}

Delaying laminar-turbulent transition in a boundary layer (BL) has been a subject of great interest over several decades, due to its fundamental engineering implications in several real flow applications. The prototype flow for this kind of investigations is the Blasius boundary layer developing on a flat plate. In this case, for low-intensity external disturbances the transition scenario is dominated by exponentially growing plane waves, known as Tollmien-Schlichting (TS) waves. It has been shown that TS waves can be attenuated by properly shaped and stable spanwise velocity modulations inside the BL, denoted streaks, thus leading to transition delay. The streaks are high- and low-speed regions in the boundary layer generated by a convective mechanism transporting fluid with high momentum close to the wall and ejecting low-momentum fluid in the outer region of the BL. This process is usually referred to as the lift-up mechanism (Landahl 1980). 
To the authors' knowledge, the first experimental evidence of stabilization of TS waves by streamwise streaks is reported in Kachanov \& Tararykin (1987). A theoretical support has been proposed in Cossu \& Brandt $(2002,2004)$ by direct numerical simulation (DNS) and local temporal stability analysis. In the experiments in Fransson et al. (2004, 2005, 2006), where streamwise velocity streaks are generated using small circular roughness elements installed on the plate surface, a systematic study is dedicated to investigate the effects of velocity streaks on the dynamics of TS waves and on transition. It was shown that higher streak amplitudes improved the stabilization up to a certain threshold amplitude, above which a by-pass transition at the location of the roughness elements occurred. In order to increase the maximum amplitude of the generated stable streaks, which is around $12 \%$ of the free-stream velocity $\left(U_{\infty}\right)$ when circular roughness elements are used, Fransson \& Talamelli (2012) proposed to employ small winglet pairs periodically distributed in the spanwise direction, denoted miniature vortex generators (MVGs). It is shown experimentally that MVGs can generate maximum streak amplitudes up to $32 \%$ of $U_{\infty}$. The MVGs generate streamwise vortices, which modulate the BL and form velocity streaks by the lift-up mechanism. The streamwise decay of the streaks can be quite rapid, since the generated vortices lie inside the boundary layer where viscous effects are important. To overcome this problem, Fransson \& Talamelli (2012) proposed to use multiple MVG arrays distributed in the streamwise direction so as to regenerate the streaks and to extend the stabilizing effect further downstream. The possibility to delay transition and accomplish net skin-friction drag reduction of at least $65 \%$ using MVGs has been investigated experimentally and reported in Shahinfar et al. (2012, 2013, 2014) and Sattarzadeh et al. (2014).

In order to avoid the installation of devices inside the boundary layer, which can be detrimental for stability in off-design flow conditions, and to create streaks with a strong persistency in the streamwise direction, it would be advantageous if MVGs or similar devices generating streamwise vortices could be placed outside the boundary layer. In this way, it would be possible to relax the limitations due to the scaling with the boundary layer thickness, decrease viscous damping and avoid geometric discontinuities in the boundary layer. These considerations motivate the present work, which is dedicated to investigate numerically the generation of velocity streaks by utilizing the lift-up mechanism through free-stream vortices, which penetrate the BL further downstream, and to characterize the effect of the generated streaks on the stability of the boundary layer itself. The work is inspired by the experiment documented in Tani \& Komoda (1962), where small amplitude streamwise streaks $(\simeq 6 \%$ of the freestream velocity) were produced with a series of small wings placed outside the boundary layer. In the present work we do not focus on the design of a specific device to generate free-stream vortices, as for instance the winglets used in Tani \& Komoda (1962), instead we directly simulate the evolution of idealized vortices in the flow, varying the free parameters of the flow configuration in a range of realistic values according to our previous experience with the DNS of the flow past MVGs (Camarri et al. 2013), but without parameter optimization. Our result is a proof-of-concept that it is possible to identify configurations of free-stream vortices, which show very promising characteristics and hence will be worth testing experimentally with free-stream turbulence. Among the tested configurations two are selected and investigated in more detail here.

\section{Flow configuration}

A sketch of the considered flow configuration is shown in figure 1 with ideal freestream vortices being introduced at a given distance from the plate leading edge $\left(X_{\mathrm{v}}\right)$. 


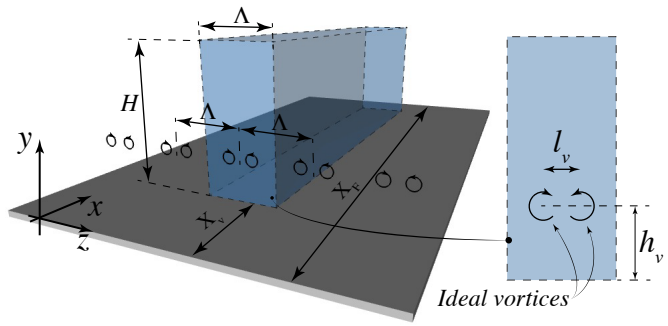

FiguRE 1. Sketch of flow configuration.

The vortices are placed outside the boundary layer at a distance $h_{\mathrm{v}}$ from the wall and have a radius $r_{0}$ of the same order of magnitude as the BL thickness. The spanwise periodicity of the vortex pairs is denoted $\Lambda$. Two different vortex models have been used to approximate the vorticity inside each single vortex, i.e. the Rankine- and the Batchelor vortex model (see Batchelor 1964). In both models the vortices are assumed perfectly circular with total circulation $\Gamma_{0}$. In the Rankine model axial vorticity is uniform inside the cores, so that the induced velocity field is tangential to the core and its intensity increases linearly inside the core and decreases as $1 / r$ outside, $r$ begin the distance from the vortex center. In the Batchelor vortex adopted here (?) the induced velocity has both a tangential $\left(v_{\theta}\right)$ and an axial $\left(v_{x}\right)$ :

$$
v_{\theta}(r, \theta)=\frac{\Gamma}{2 \pi r}\left[1-\exp \left(-\frac{r^{2}}{r_{0}^{2}}\right)\right], \quad v_{x}(r, \theta)=-\frac{\Gamma}{2 \pi l_{p}}\left[1-\exp \left(-\frac{r^{2}}{r_{0}^{2}}\right)\right],
$$

where $l_{p}$ is the helical pitch of the vortex which determines the velocity defect in streamwise direction. In order to choose reasonable ranges to vary the free parameters of the considered flow configuration we have selected a successful experimental configuration, namely the C01 case documented in Shahinfar et al. (2013), as guidance. The vortices, inducing streaks in the C01 case, were generated using MVGs mounted on the surface and has been studied in detail using DNS (Camarri et al. 2013), which makes this case suitable. In (2.1) C01 the fluid is air at atmospheric conditions, the free-stream velocity was $U_{\infty}=7.7 \mathrm{~m} \mathrm{~s}^{-1}$, the kinematic viscosity was $\nu=1.46 \times 10^{-5} \mathrm{~m}^{2} \mathrm{~s}^{-1}$, the height of the MVGs were $1.3 \mathrm{~mm}$ and were installed $222 \mathrm{~mm}$ downstream of the leading edge of the plate. The streamwise position was chosen such that the maximum amplitude of the generated streaks was close to branch I of the stability curve for the uncontrolled case without MVGs. In the present paper we consider the same flow parameters $U_{\infty}$ and $\nu$, and our results are sometimes shown in dimensional form to facilitate the comparison with the experimental C01 case.

\section{Numerical setup}

\subsection{Direct numerical simulations}

The numerical code used for the DNS simulations is NEK5000, an open-source massively parallel spectral element code. The spatial discretization is based on a local tensorproduct of Gauss-Lobatto Legendre (GLL) quadrature points within each hexahedral element. Pressure stabilization is obtained by a $P_{\mathrm{N}}-P_{\mathrm{N}-2}$ formulation. A third order scheme (BDF3) in time is used, employing an explicit backward-differentiation for convective terms and an implicit scheme for viscous terms. Details on the code can be found following the link http://nek5000.mcs.anl.gov/index.php/Main_Page .

The simulations of the steady boundary layer flow including the free-stream vortices 
were carried out with a computational domain starting at $X_{\mathrm{v}}$, being a variable in the range $0 \leqslant R e_{X_{v}} \leqslant 2.74 \times 10^{4}$, and ending at $X_{\mathrm{f}}$. The height of the computational domain is $H=86 \mathrm{~mm}$, giving a $H / \delta \geqslant 274$ at the inlet section where $\delta(x)=\sqrt{\nu x / U_{\infty}}$ is the characteristic length scale of the BL. The simulations are limited to computing only one pair of free-stream vortices, i.e. with a spanwise computational domain width of $1 \Lambda$, but with imposing periodic boundary conditions in the spanwise direction. Two different streamwise extents of the computational domains were used: the first domain (D1) extends for a normalized length equal to $\Delta R e_{X}=R e_{X_{\mathrm{f}}}-R e_{X_{\mathrm{v}}} \simeq 4.24 \times 10^{5}$ and employs about $6 \cdot 10^{7}$ degrees of freedom (dofs); the second one (D2), used for the configurations of slowly evolving streaks, has $\Delta R e_{X}=R e_{X_{\mathrm{f}}}-R e_{X_{\mathrm{v}}} \simeq 1.00 \times 10^{6}$ with about $10^{8}$ dofs.

The imposed velocity distribution at the inflow at $X_{\mathrm{v}}$ consists of a superposition of the velocity field induced by a pair of free-stream vortices of different types to a Blasius boundary layer profile. This implies that the axis of the vortices at $X_{\mathrm{v}}$ is parallel with the plate. No-slip boundary conditions are used at the solid wall and null-stress outflow boundary conditions, i.e $P-R e^{-1} \partial U / \partial x=0, \partial V / \partial x=0 \partial W / \partial x=0$, are imposed on the outflow and on the top boundary. The spatial resolution has been chosen on the basis of the simulations reported in Camarri et al. (2013) and of dedicated convergence tests which are not shown here for the sake of brevity. Moreover, the results of the DNS simulations have been further validated against those obtained by a 3D boundary layer solver written on purpose and employing a completely different numerics (mixed finite-differences/finite-elements discretization on unstructured grid).

Simulations of transition, triggered by forcing high initial amplitude TS waves, have also been performed in order to investigate the control effect by the free-stream vortices on transition delay. In these simulations the domain was extended to $10 \Lambda$ in the spanwise direction, thus including 10 pairs of counter-rotating vortices avoiding any influence of the boundary conditions on the transition location. In the streamwise direction the domain extends from $R e_{X_{\mathrm{v}}}=5.28 \times 10^{4}$ to $R e_{X_{\mathrm{f}}}=6.11 \times 10^{5}$ and the normalized forcing frequency of the TS wave is $F=10^{6}\left(2 \pi f \nu / U^{2}\right)=140$. The TS waves are excited in the DNS by a time-periodic plane volume force, localized at point at $X_{\mathrm{TS}}$. The adopted boundary conditions are the same as for the simulation of the base flow. Concerning the spatial discretization, convergence tests have been carried out leading to a final grid with about $2 \cdot 10^{8}$ dofs.

\subsection{Biglobal stabiltiy analysis}

The linear stability of the streaky BL induced by the free-stream vortices has been characterized by performing a local biglobal stability analysis, similar to the analysis by Piot et al. (2008) on the flow past a periodic row of roughness elements. Since the flow is fully three-dimensional, the discretization of the stability problem has to be performed in the cross-sectional $y z$-plane at each downstream location. The local stability problem is governed by the linearized Navier-Stokes equation (LNSE) for an incompressible flow and a generic solution, of the streamwise $(u)$, wall-normal $(v)$, and spanwise $(w)$ velocity disturbances as well as the pressure perturbation $(p)$, is searched for in the following modal form: $\{u, v, w, p\}=\{\tilde{u}, \tilde{v}, \tilde{w}, \tilde{p}\}(y, z) e^{i(\alpha x-\omega t)}$. Here, $\sim$ denotes the disturbance amplitude distribution in the $y z$-plane, $\alpha$ is the streamwise wavenumber and $\omega$ the angular frequency. The disturbance is assumed to vanish on and far away from the wall, and to be periodic in the spanwise direction (sub-harmonic modes have also been checked using multiples of the periodicity length of the base flow). When the LNSE are discretized in space, a generalized eigenvalue problem is obtained, which is quadratic in $\alpha$ for the spatial analysis ( $\omega$ is fixed) and linear in $\omega$ in the temporal analysis ( $\alpha$ fixed). Spatial discretiza- 

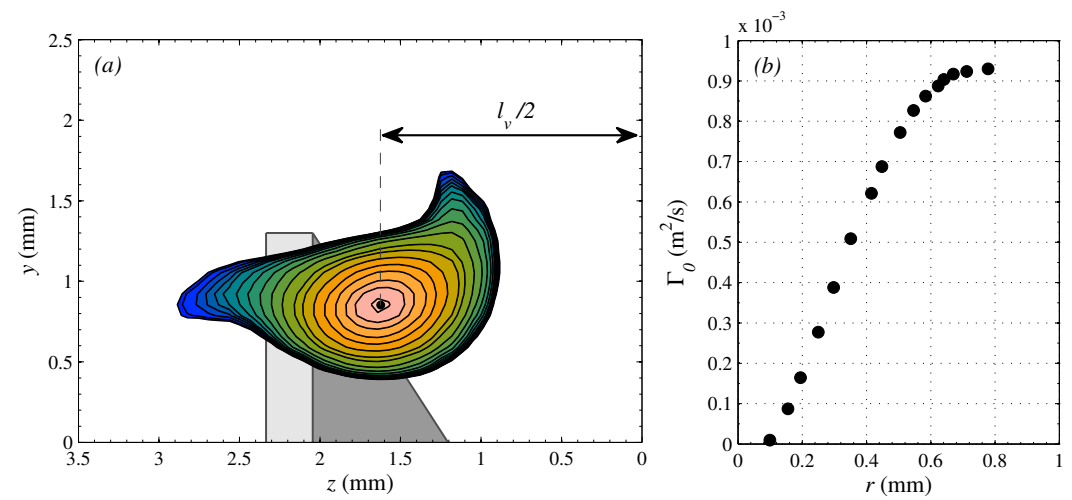

Figure 2. (a) Streamwise vorticity in the vortex past one MVG (shown in background) for configuration $\mathrm{C} 01$ at a distance $13 \mathrm{~mm}$ past the MVG (isocontours equally distributed from 0 to $1.0 \cdot 10^{-3} \mathrm{~m}^{2} / \mathrm{s}$ ) and (b) circulation computed on the iso-contours of vorticity plotted against the area-weighted mean distance $r$ of the isocontour from the vortex center.

tion is here obtained by a finite-element formulation on unstructured grids, employing Taylor-Hood elements. The open-source code FreeFem++ (http://www.freefem.org) has been used to this purpose. The stability has been characterized by a spatial stability analysis. The initial guess for the solution of the resulting non-linear eigenvalue problem has been estimated performing a temporal stability analysis and applying the Gaster transformation to the results. Both the linear and non-linear eigenvalue problems have been solved by a Krylov-Schur method with a shift-invert technique, using the parallel implementation available in the SLEPc library (see ????).

\section{Results and discussion}

4.1. Exploration of the free parameters and selection of a reference case

The considered flow depends on the free parameters detailed in Sec.2. With reference to Figure 1 the geometric parameters are $X_{v}, h_{v}, l_{v}, \Lambda$, while each vortex is characterized by $r_{0}, \Gamma_{0}$ and, for the Batchelor vortex model, $l_{p}$. As already discussed, a rigorous optimization or even a refined exploration of all the free parameters would imply large computational costs. At the same time, the objective of the present paper is to show that, even without optimization, it is possible to identify flow configurations with promising characteristics in terms of streaks evolution and of flow stability. Moreover, since vortices are introduced in an idealized form, it is important to grant the physical realizability of the flow by varying the free parameters within physically meaningful ranges. These have been determined on the basis of previous experience with MVGs. In particular, a reasonable initial guess for $r_{0}, \Gamma_{0}$ and $l_{v}$ has been found by analysing the details of the vorticity past the MVGs in the configuration C01 (see ?), extracted from the DNS data in ?. In this respect, Figure 2(a) shows the streamwise vorticity inside one of the two counter-rotating vortices past a couple of MVGs at a downstream distance of $13 \mathrm{~mm}$ past the MVG. Circulation of velocity has been computed on circuits coinciding with iso-contours of axial vorticity reported in Figure 2(a) and the data has been collected in Figure 2(b), where circulation is plotted against the average distance $r$ of each isocontour from the center of the vortex. The synthetic data in Figure 2(a-b) have been used to derive a plausible value for $r_{0}, l_{v}$ and $\Gamma_{0}$, those being equal to $0.8 \mathrm{~mm}, 3.12 \mathrm{~mm}$ and $0.910^{-4} \mathrm{~m}^{2} / \mathrm{s}$, respectively. The parameter $l_{v}=3.12 \mathrm{~mm}$ has been kept constant for all 

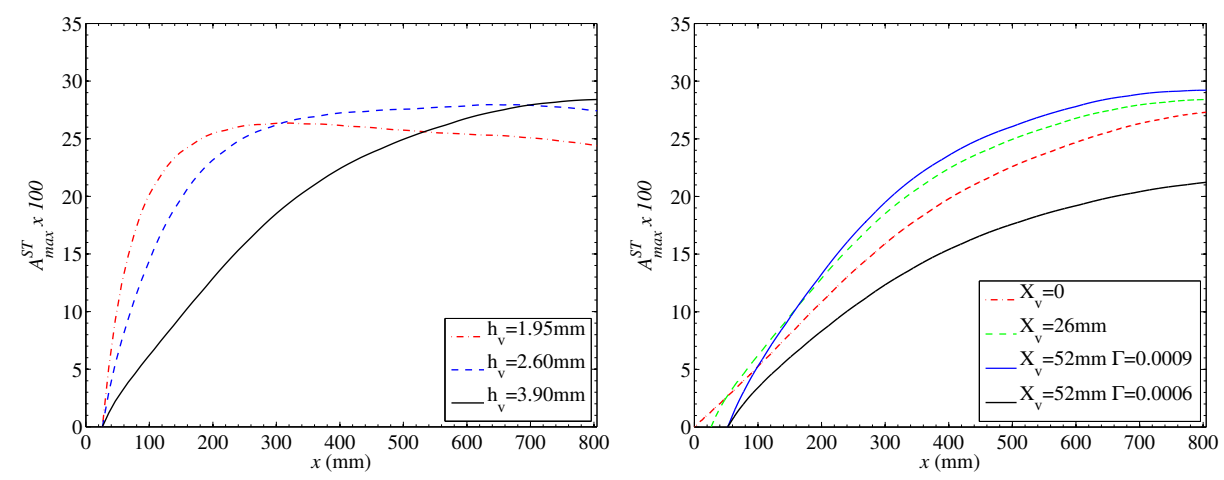

FiguRE 3. Evolution of the streak amplitude in the streamwise direction varying (a) $h_{v}$ $\left(X_{v}=26 \mathrm{~mm}\right)$ and $(\mathrm{b}) X_{v}\left(h_{v}=3.90 \mathrm{~mm}\right)$ (Rankine model is used for the idealized vortices).

the considered cases. Also the spanwise spacing of the pairs of vortices, $\Lambda=13 \mathrm{~mm}$, has been kept constant and equal to that of the MVGs in C01. The parameters $X_{v}$ and $h_{v}$ are the only ones which cannot be guessed on the basis of case $\mathrm{C} 01$ because they are related to the placement of an hypothetical device generating the vortices. The constraint on $h_{v}$ is to generate the vortices mostly outside the boundary layer and $X_{v}$ should be chosen in order to obtain an appreciable streak amplitude in the region of convective instability for the uncontrolled BL.

On the basis of the considerations above, an exploratory set of simulations have been carried out using domain D1 and varying the free parameters in the following ranges: $0.65 \mathrm{~mm} \leqslant r_{0} \leqslant 1.3 \mathrm{~mm}, 1.30 \mathrm{~mm} \leqslant H \leqslant 7.80 \mathrm{~mm}, 0 . \mathrm{mm} \leqslant X_{v} \leqslant 235.00 \mathrm{~mm}$, $6 \cdot 10^{-4} \mathrm{~m}^{2} / \mathrm{s} \leqslant \Gamma_{0} \leqslant 27 \cdot 10^{-4} \mathrm{~m}^{2} / \mathrm{s}$. A representative subset of the obtained results, using the Rankine model for the inlet conditions, is reported in Figure 3, where the amplitude $A_{\max }^{S T}$ of the generated stable streaks is shown in terms of $x, A_{\max }^{S T}$ being defined as follows: $A_{\max }^{S T}=\max _{y}\left\{\max _{z}\{U(X, y, z)\}-\min _{z}\{U(X, y, z)\}\right\} /\left(2 U_{\infty}\right)$. Figure 3(a) highlights the effect of the sole $h_{v}$ on the generated streaks $\left(X_{v}=26.0 \mathrm{~mm}\right.$.) Three values of $h_{v}$ are considered, i.e. $h_{v}=1.95,2.60,3.90 \mathrm{~mm}$, corresponding to $h_{v} / \delta\left(X_{v}\right)=8.8,11.7,17.6$. As the value of $h_{v}$ is increased, the vortices are progressively more distant from the BL so they are subjected to a lower dissipation rate and, at the same time, they induce a lower velocity on the BL. Consequently, as shown in Figure 3(a), as $h_{v}$ is increased the maximum amplitude of the streaks moves in downstream direction and slightly increases. The effect of a variation of $X_{v}$ is reported in Figure 3(b), showing that results are not significantly sensitive for the considered range of variation of $X_{v}$. The slight differences among the cases in Figure 3(b) might be explained considering that, besides $X_{v}$ is varying, the relative position between the vortices and the external boundary of the BL, $h_{v} / \delta\left(X_{v}\right)$, also varies because $\delta\left(X_{v}\right)$ increases with $X_{v}, h_{v}$ being constant. Figure 3(b) also reports a case with a lower value of $\Gamma$, namely $\Gamma=6 \cdot 10^{-4} \mathrm{~m} / \mathrm{s}$ (black line). As expected, the evolution of the streaks is very similar to the corresponding case with higher $\Gamma$ (blue line), but amplitude is of course lower and scales almost linearly with $\Gamma$. Among the cases in Figure 3, in the following part of the paper we focus on the cases reported with blue (E1, $\left.r_{0}=1.3 \mathrm{~mm}, H=3.9 \mathrm{~mm}, \Gamma_{0}=9 \cdot 10^{-4} \mathrm{~m}^{2} / \mathrm{s}, X_{v}=52 \mathrm{~mm}\right)$ and black $(\mathrm{E} 2$, as E1 but with $\Gamma_{0}=6 \cdot 10^{-4} \mathrm{~m}^{2} / \mathrm{s}$ ) lines in Figure $3(\mathrm{~b})$, whose results are reported for a longer streamwise extension in Figure 5(a). Among those, our reference case will be E2. This has been designed so as to obtain a maximum amplitude of the generated streaks approximately equal to that in $\mathrm{C} 01$, which was shown to be an optimal value to delay TS waves instability and transition. Indeed, as shown in the following, higher values of 
streak amplitude, as in E1, may lead to an instability of the flow, even if significantly delayed with respect to the uncontrolled case.

Finally, as concerns the sensitivity to the model used for the idealized vortices, the case E1 has been computed also using the Batchelor model (case E1b). In this case the helical pitch $l_{p}$ has been set equal to $2.6 \mathrm{~mm}$, in agreement with the experimental results in ?, which are focused on the evolution of a pair of counter-rotating vortices in the wake past a rectangular winglet placed closed to a plane wall, this being a very similar configuration to that investigated here. Results for E1b are also reported in Figure 5(a), where it is highlighted that the differences in streak amplitude among the two different vortex models lead to a slight discrepancy, which is at maximum of $2 \%$ in terms of $A_{\max }$. Thus, we can conclude that results obtained are moderately sensitive to the model selected to represent the idealized vortices.

\subsection{Streaks streamwise evolution}

In this section we focus on the characteristics of the streaks generated in the reference case E2. Figure 4 shows the streamwise velocity (colormap) together with the streamwise vortices, identified using iso-contours of the $\lambda_{2}$ parameter, at thee different distances from the plate leading edge. The three plots clearly show that the streamwise vortices induce a velocity field in $x-z$ planes which tends to drive high momentum fluid from the external flow between the two vortices of the pair into the BL, while low momentum flow is liftedup in the region between two contiguous vortex pairs. Since $l_{v}<\Lambda$, the resulting high velocity streaks are narrower and stronger than the low velocity ones. Moving along the plate, the streamwise vortices progressively approach and enter in the boundary layer. At the same time, they loose their intensity when moving in the downstream direction, as quantified in Figure 6(a) (blue line) where the streamwise evolution of the circulation $\Gamma_{0}$ is reported for both E1 and E2 cases.

The amplitude $A_{\max }^{S T}$ measured experimentally for the case $\mathrm{C} 01$ is also reported in Figure 5 for comparison, showing that when MVGs are used, the streaks take a definitely shorter distance to form since the vortices are intense and inside the BL, but the streaks also dissipate more rapidly. When vortices equivalent to those past the MVGs are positioned outside the boundary layer (case E1), they generate streaks which are more intense because they induce a lift-up mechanism for a longer distance. Since, however, the induced velocities are lower due to a larger distance from the wall, the generation process takes a longer distance in the streamwise direction to take place. Thus, to obtain a maximum amplitude equal to that in case C01, less intense vortices can be used, as in case E2. For a further comparison between the case E2 and C01, Figure 5(b) shows the streamwise velocity and the streamwise vortices of E2 (left) and of C01 (right) at the point of maximum streak amplitude of both cases. The figure well highlights the differences between the two cases, especially in the shape of the velocity streaks, and clearly shows the different position of the streamwise vortex with respect to the boundary layer.

\subsection{Effect on boundary layer stability}

In this section we present the results of the bi-global spatial stability analysis applied to the cases E1 and E2. The results are condensed in the stability curve (blue line) reported in the $F-R e$ plane in Figure 6(b), where the corresponding curve for the uncontrolled Blasius boundary layer is also reported (dashed line) for comparison between the controlled and the uncontrolled cases. Please note that Figure 6 reports only the stability curve (blue line) for the case E1 because the flow field generated in case E2 is stable for all the sections of the computational domain. Thus, in E2 the linear instability of the 

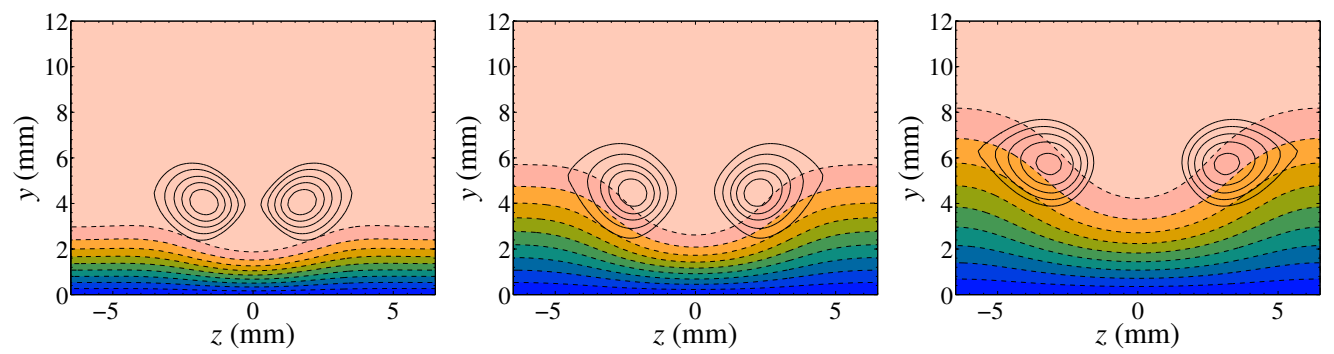

Figure 4. Case E2: cross-sectional contours of mean streamwise velocity field $U$, normalized with the free-stream velocity $U_{\infty}$ : (a) $x=310 \mathrm{~mm}$, (b) $x=850 \mathrm{~mm}$ and (c) $x=1675 \mathrm{~mm}$. The dashed black lines are contours of constant velocity (.1:.1:.9). The solid black lines represent the $\lambda_{2}$ vortex identification criterion.
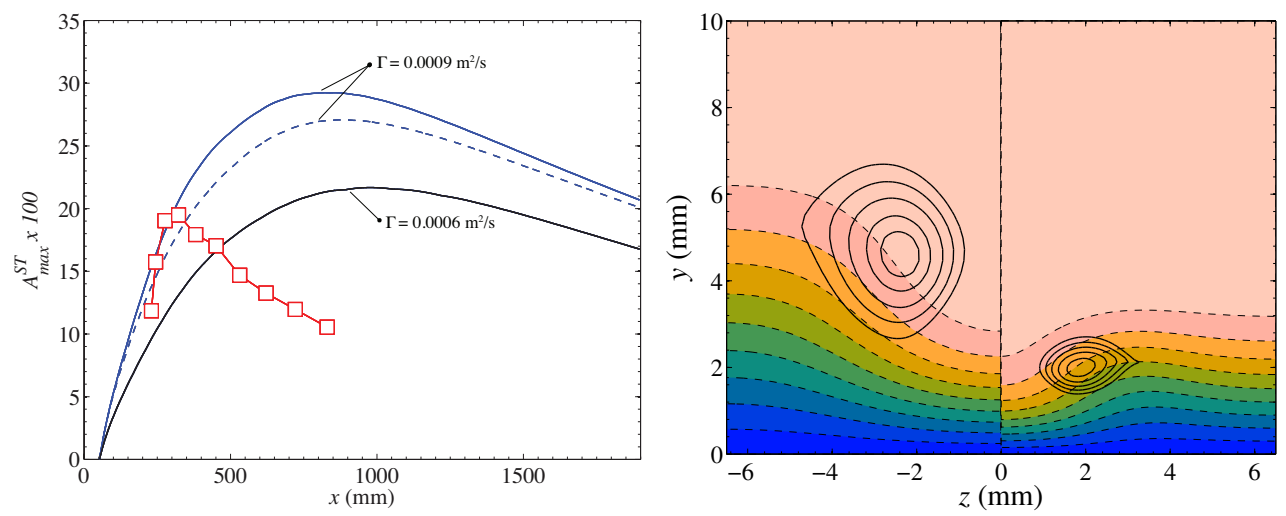

FiguRE 5. (a) Streaks amplitude evolution in streamwise direction for the MVGs' case C01 ( $\square$, from experiments), for the case E1 (blue line), E1b (dashed line) and E2 (black line); (b) crosssectional contours of mean streamwise velocity field $U$, normalized with the free-stream velocity $U_{\infty}$, for E2 (left) and for C01 (DNS, right) at the position of maximum streaks amplitude. The solid black lines represent the $\lambda_{2}$ vortex identification criterion.

$\mathrm{BL}$ is delayed at least up to a distance of $1.5 \cdot 10^{3} \mathrm{~mm}$ from the leading edge of the plate. As concerns the case E1, the beginning of the instability region is moved significantly downstream if compared with the Blasius neutral curve. Inside the instability region, the growth factors for both cases are comparable (not shown in the figure), indicating that also the E1 controlled case is a potentially attractive configuration for transition delay. However, while in E2 the flow is always stable, in E1 instability starts at the streamwise position where the maximum amplitude of the streak is reached (see Figure 5). This fact, the shape of the unstable mode and the knowledge from the literature on maximum amplitudes for streak stability suggest that the onset of instability in E1 is driven by an instability of the streaks themselves, whose amplitude is too high. This explains also why in E2, where the streaks amplitude is lower, the convective instability of the boundary layer is completely stabilized for all the length of the computational domain. Moreover, results presented here demonstrates that there is a large margin of improvement for the stability of the controlled BL because it is possible to modify the streak amplitude by acting on several parameters. For instance, amplitude of the generated streaks can be controlled not only acting on $\Gamma$, as done switching from E1 to E2, but also modifying the initial distance $h_{v}$ of the vortices from the wall, so as to reduce the intensity of the induced velocities leading to the lift-up mechanism. However, even in the present configurations E1 and E2, the delay in the onset of convective instability inside the BL is 

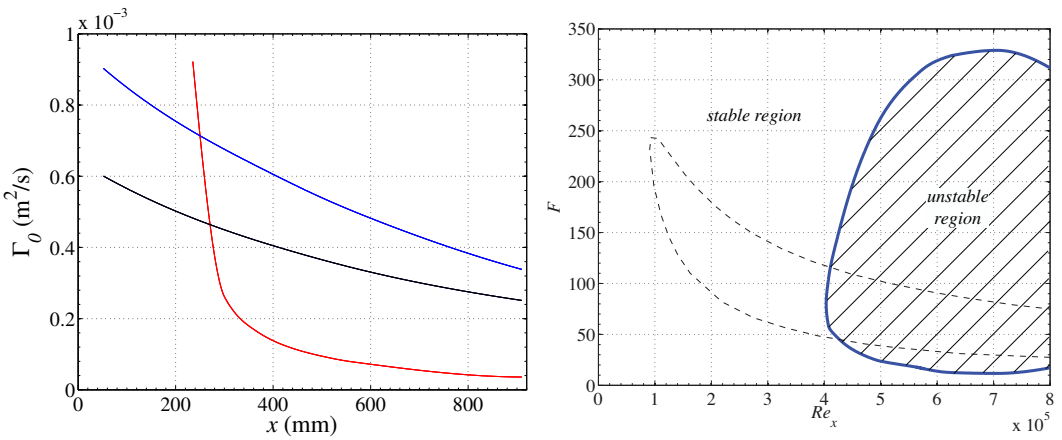

FiguRE 6. (a) Streamwise evolution of the circulation of the streamwise vortices for E1 (blue line), E2 (black line) and for C01 (DNS, red line); (b) in solid blue line the neutral curve for E1 (blue line) in comparison with the neutral curve for Blasius boundary layer (dashed line) in $F-R e$ plane; case E2 is stable in the range of $R e_{x}$ considered in the figure.

so evident that a gain in terms of transition delay is expected by the proposed control. This is shown in the next section.

\subsection{Boundary-layer transition delay}

Experiments and DNS simulations have been carried out for the case C01 forcing transition with an excitation at normalized frequency $F \simeq 140$. This frequency was selected for $\mathrm{C} 01$ because the position of Branch I of the stability curve in the uncontrolled case was very closed to the region where the maximum streaks amplitude generated by the MVGs was reached. In the DNS documented here the TS waves are forced as detailed in Section 3 at $F=140.6$. The forcing has been introduced in a DNS simulation of the uncontrolled Blasius boundary layer, and the forcing amplitude has been varied and tuned so as to observe transition inside the computational domain. This is shown in Figure 7(a), where the temporal root mean square fluctuations of the normalized streamwise velocity $\left(u_{r m s} / U_{\infty}\right)$ is plotted at a distance of $1.0 \mathrm{~mm}$ from the wall. The figure clearly shows that the excitation is introduced at a streamwise position $x_{f}=150 \mathrm{~mm}$, TS waves encounter Branch I where rms starts to grow up to Branch II, after which it decreases but the flow undergoes a secondary instability triggering transition after $x \simeq 750 \mathrm{~mm}$. The same forcing is thus introduced in the boundary layer controlled by the external vortices in the case E1, and the result reported in Figure 7(b) shows that the disturbance is rapidly damped after a transient initial growth. TS waves reach such a low value that it is not possible to detect, at the scale of the plot, any successive growth when they enter again in the convectively unstable region, which is almost at the end of the computational domain. The test carried out in the present section shows that, even considering the case E1, which is characterized by the stability curve illustrated in Figure 5, it is possible to show an evident transition delay with respect to the uncontrolled boundary layer, at least for the considered flow conditions and forcing frequency. An even better behavior is expected from slightly optimized configurations, as E2, where the onset of convective instability in the BL is even further delayed than in E1.

\section{Conclusions}

The authors wish to acknowledge PRACE for awarding access to resource Fermi based in Italy at Cineca. JHMF acknowledges the European Research Council for their financial support of the AFRODITE project through a Starting Independent Researcher Grant. 

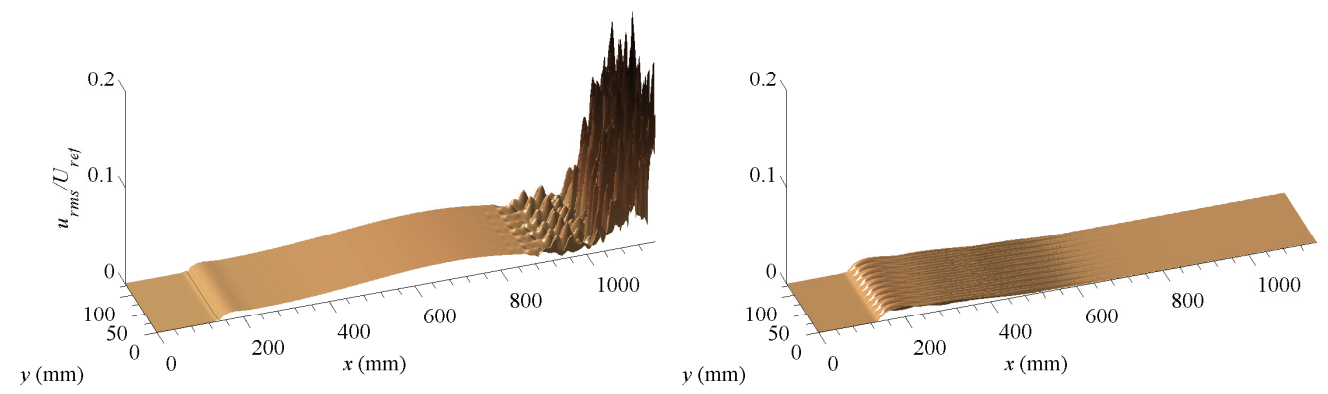

FIGURE 7. Evolution of the dimensionless temporal root mean square streamwise velocity fluctuations $u_{r m s} / U_{\infty}$ in streamwise direction at $y=1.0 \mathrm{~mm}$ (TS waves at $F \simeq 140$ ): (a) uncontrolled Blasius BL and $(b)$ controlled case E1.

\section{REFERENCES}

Batchelor, G. K. 1964 Axial flow in trailing line vortices. J. Fluid Mech. 20, 645-658.

Camarri, S., Fransson, J. H. M. \& Talamelli, A. 2013 Numerical investigation of the afrodite transition control strategy. In Progress in Turbulence $V$ (ed. A. Talamelli, M. Oberlack \& J. Peinke), pp. 65-69. Springer.

Cossu, C. \& Brandt, L. 2002 Stabilization of Tollmien-Schlichting waves by finite amplitude optimal streaks in the Blasius boundary layer. Phys. Fluids 14, L57-L60.

Cossu, C. \& Brandt, L. 2004 On Tollmien-Schlichting-like waves in streaky boundary layers. Eur. J. Mech./B Fluids 23, 815-833.

Fransson, J. H. M., Brandt, L., Talamelli, A. \& Cossu, C. 2004 Experimental and theoretical investigation of the nonmodal growth of steady streaks in a flat plate boundary layer. Phys. Fluids 16 (10), 3627-3638.

Fransson, J. H. M., Brandt, L., Talamelli, A. \& Cossu, C. 2005 Experimental study of the stabilisation of Tollmien-Schlichting waves by finite amplitude streaks. Phys. Fluids 17, 054110.

Fransson, J. H. M. \& Talamelli, A. 2012 On the generation of steady streamwise streaks in flat-plate boundary layers. J. Fluid Mech. 698, 211-234.

Fransson, J. H. M., Talamelli, A., Brandt, L. \& Cossu, C. 2006 Delaying transition to turbulence by a passive mechanism. Phys. Rev. Lett. 96, 064501.

KACHANOV, Y. S. \& TARARYKIn, O. I. 1987 Experimental investigation of a relaxating boundary layer. Izv. SO AN SSSR, Ser. Tech. Nauk 18.

LANDAHL, M. T. 1980 A note on an algebraic instability of inviscid parallel shear flows. J. Fluid Mech. 98, 243-251.

Piot, E., Casalis, G. \& Rist, U. 2008 Stability of the laminar boundary layer flow encountering a row of roughness elements: Biglobal stability approach and DNS. Eur. J. Mech. B. 27, 684-706.

Sattarzadeh, S. S., Fransson, J. H. M., Talamelli, A. \& Fallenius, B. E. G. 2014 Consecutive turbulence transition delay with reinforced passive control. Phys. Rev. E 89, 061001(R).

Shahinfar, S., Fransson, J. H. M., Sattarzadeh, S. S. \& Talamelli, A. 2013 Scaling of streamwise boundary layer streaks and their ability to reduce skin-friction drag. J. Fluid Mech. 733, 1-32.

Shahinfar, S., Sattarzadeh, S. S. \& Fransson, J. H. M. 2014 Passive boundary layer control of oblique disturbances by finite-amplitude streaks. J. Fluid Mech. 749, 1-36.

Shahinfar, S., Sattarzadeh, S. S., Fransson, J. H. M. \& Talamelli, A. 2012 Revival of classical vortex generators now for transition delay. Phys. Rev. Lett. 109, 074501.

TANi, I. \& Komoda, H. 1962 Boundary layer transition in the presence of streamwise vortices. J. Aerospace Sci. 29, 440. 